\title{
Burden of COPD among population above 30 years in India: protocol for a systematic review and proposed meta-analysis
}

\author{
Nachiket Gudi ${ }^{1}$, Amreen Mahmood ${ }^{2}$, Manas Pratim Roy ${ }^{3}$, Ravishankar ${ }^{4}$, Pradeepa Nayak ${ }^{5}$, Ashwani Verma $^{6}$
}

\begin{abstract}
N Gudi, A Mahmood, M P Roy, Ravishankar, P Nayak, A Verma. Burden of COPD among population above 30 years in India: protocol for a systematic review and proposed meta-analysis. Can J Respir Ther 2021;57:14-17. doi: 10.29390/cjrt-2020-040.

Background: The Sustainable Development Goals and the National Health Policy of India aim to reduce premature mortality from noncommunicable diseases (NCD) by one-third in the next decade and by 25\% by 2025, respectively. Among NCDs globally, chronic obstructive pulmonary disease (COPD) is a major contributor to death and disability. This underscores the need to understand the burden of COPD at the national level by synthesizing evidence and collating the state-wise COPD data to estimate the prevalence of COPD and to highlight the associated risk factors to inform policymakers.

Method: The systematic literature search will be carried out in PubMed, Cochrane, Scopus, Web of Science, CINAHL, and ProQuest databases with restrictions for studies published between 2000 and 2020 and available in English. Cross-sectional or cohort studies conducted in and among the Indian population aged 30 years and above will be included. Case reports, randomized trials, meta-analysis, commentaries, and qualitative studies will be excluded from the review. Quality assessment of the included studies will be performed using New Castle Ottawa scale and adherence to reporting standards will be checked using STROBE checklist for Observational Cohort and Cross-Sectional Studies.

Discussion: Prevalence of COPD in the population aged 30 years and above, diagnosed through spirometry and nonspirometry, will be compared and reported and a meta-analysis will be performed to obtain pooled prevalence rates of COPD and the risk factors associated with COPD.
\end{abstract}

Key Words: COPD; chronic bronchitis; India; systematic review; meta-analysis

\section{INTRODUCTION}

According to the Global Burden of Disease study (2017) [1], between 1990 and 2017, there was a global reduction in mortality due to maternal and neonatal disorders, enteric infections, and tuberculosis and a rise in deaths due to noncommunicable diseases (NCDs). As reported in the study, the leading causes of early deaths among NCDs are ischaemic heart disease, stroke, and chronic obstructive pulmonary disease (COPD) [1]. COPD is a common, preventable, and treatable NCD characterized by persistent respiratory symptoms and airflow limitation due to abnormalities in the airway and (or) alveoli [2]. The study [1] reported that in 2016, worldwide cases of COPD were over 250 million. From 1990 to 2015, the global prevalence of COPD increased by $44.2 \%$ [3]. In a systematic review and meta-analysis, Halbert et al. [4] reported the pooled prevalence of COPD from 37 studies is $7.6 \%$, and pooled prevalence from 26 spirometry estimates is $8.9 \%$. Meta-analysis estimates from 123 globally searched studies reported the COPD prevalence rates among people aged 30 years and older is $11.4 \%$ [5].

In 2015, nearly 3.2 million global deaths were reported due to COPD (i.e., $5 \%$ of overall deaths) with an increase of $11.6 \%$ in deaths due to COPD as compared to 1990. Globally in 2015, 63.9 million disability-adjusted life years (DALYs), which is defined as the sum of years of potential life lost due to premature mortality and the years of productive life lost due to disability [6], were reported due to COPD.
This represented $2.6 \%$ of the entire global burden of disease [3]. Along with DALYs, COPD also affects the health-related quality of life of COPD patients. Among COPD patients, quality of life deteriorates with the progression of disease due to a decline in lung function, worsening symptoms, and presence of risk factors, which leads to gradual impairment of physical performance [7, 8]. Besides disease burden, COPD contributes to the economic burden in terms of direct and indirect costs related to disease management. Working-age group (18-65 years) patients with COPD have higher direct and indirect costs and were more resource-intensive [9].

Previous evidence revealed an overall prevalence of COPD in low- and middle-income countries (LMICs) was 9.2\% (1.2\% to $15.4 \%$ ) [10]. Meta-analysis estimates found the prevalence of COPD among patients 30 years and older was 10.6 in LMICs [5]. More than $90 \%$ of COPD-related deaths occur in LMICs [11]. India has a population of nearly 1.2 billion across 36 states and union territories [12]. Few studies have been conducted in India to report the disease burden due to $\mathrm{COPD}$, and the prevalence of COPD varies widely, representing a large public health problem. The global burden of disease study for India reported the prevalence of COPD increased from 3.3\% (28.1 million cases) in 1990 to $4.4 \%$ (55.3 million cases) in 2016 [13].

COPD is the second most common cause of NCD-related deaths in India, with the age-specific prevalence of COPD increasing rapidly after

${ }^{1}$ Department of Health Policy, Prasanna School of Public Health, Manipal Academy of Higher Education, Manipal, Karnataka, India; ${ }^{2}$ Department of Physiotherapy. Kasturba Medical College, Mangalore, Manipal Academy of Higher Education, Karnataka, India; ${ }^{3}$ Deputy Assistant Director General (NCD), Directorate General of Health Services, Nirman Bhawan, New Delhi, India; ${ }^{4}$ Department of Data Science, Prasanna School of Public Health, Manipal Academy of Higher Education, Manipal, Karnataka, India; ${ }^{5}$ Department of Physiotherapy, Manipal College of Health Professions, Manipal Academy of Higher Education, Manipal, Karnataka; ${ }^{6}$ Evidence Synthesis Specialist, Campbell South Asia, Global Development Network, 2 nd Floor, West Wing, ISID Complex, Plot No4, Vasant Kunj Institutional Area, Vasant Kunj, New Delhi, Delhi 110070, India; PhD Research Scholar, DIT University, Dehradun, Uttarakhand, India

Correspondence: Dr. Ashwani Verma, Evidence Synthesis Specialist, Campbell South Asia, Global Development Network, 2nd Floor, West Wing, ISID Complex, Plot No 4, Vasant Kunj Institutional Area, Vasant Kunj, New Delhi, Delhi 110070, India. Tel: +919611496374, E-mail: vamit1989@gmail.com

Published online at https://www.cjrt.ca on 1 February 2021

OPEN ACCESS

This open-access article is distributed under the terms of the Creative Commons Attribution Non-Commercial License (CC BY-NC) (http:// creativecommons.org/licenses/by-nc/4.0/), which permits reuse, distribution and reproduction of the article, provided that the original work is properly cited and the reuse is restricted to noncommercial purposes. For commercial reuse, contact editor@csrt.com 
the age of 30 years. It was noted that the prevalence of COPD among individuals between 5 and 29 years ranged from $0.1 \%-0.9 \%$, which increased to $1.6 \%-28.3 \%$ among the population aged 30 years or more [13]. From 2007 to 2017, the percentage change in death and premature death due to COPD is over $39 \%$ and $37 \%$, respectively [14]. In India, COPD is the second most leading cause of DALYs with the $36 \%$ mean percentage change in the number of DALYs from 1990 to 2016 [15]. The rate of DALYs per case due to COPD was 1.7 times higher than the global average in 2016 [13]. Previous research studies from India suggest the economic impact in terms of direct and indirect cost is on the higher side (direct medical cost: Rs. 29,885 $\pm 11,995.33$ or US $\$ 300-500$ approximately; direct nonmedical cost: Rs. 7,441.25 $\pm 2,228.90$ or US\$90-155 approximately) and is associated with absenteeism at the job for a significant duration of time [16, 17]. A study conducted on COPD patients by Mahmood et al. in Uttar Pradesh reported that $56.5 \%$ of patients were nonsmokers, indicating the major role of second-hand smoke and other risk factors [18].

A field survey was conducted among 44,387 individuals in Bangalore and reported a $4.36 \%$ prevalence of COPD and found an increasing trend of COPD as individuals grow older [19]. Another cross-sectional study was conducted among 1200 adults and reported a $10 \%$ prevalence rate of COPD in Delhi [20]. Moreover, in 2001 Jindal et al. reported a huge variation in the prevalence of COPD ranging from $2 \%$ to $22 \%$ in men and $1.2 \%$ to $19 \%$ in women through population-based studies across India [21]. Evidence from the studies done among women in 24 villages of central India reported a $2.7 \%$ prevalence of chronic bronchitis and variation amongst social determinants of health (e.g., age, socioeconomic status, and exposure to pollution) [22]. A communitybased survey conducted in Kerala identified a $6.19 \%$ prevalence of chronic bronchitis and a $13.5 \%$ prevalence among the individuals aged above 35 years in Trivandrum district [23].

Based on the Global Initiative for Obstructive and Lung Diseases (GOLD) guideline, tobacco smoking, indoor and outdoor air pollution, occupational exposure, age, gender, and socioeconomic status are some of the risk factors for COPD [2]. Evidence from numerous research studies conducted at the state and district level supports that low socioeconomic status, occupational exposure to environmental tobacco smoke, exposure to air pollution (outdoor and indoor) are the major factors significantly associated with COPD [13, 18-21]. Biomass fuels are also considered a common cause of COPD in LMICs. Nearly 2 billion people in the world's population are exposed to biomass fuel, in which 1 billion people are exposed to outdoor air pollution, and 1 billion are either exposed to or are at risk of second-hand smoke [24]. Exposure to biomass fuel in terms of animal dung, wood, and crops, is common in developing countries like India, and nearly half of the world's households use biomass fuel. As per the 2010 census, more than $65 \%$ of the Indian population resides in rural areas. A significant population in developing countries bears a great share of disease burden due to chronic exposure to biomass fuel. Many of these activities are being practiced in an area where effluent is released into the living area $[25,26]$.

Currently, there exist studies that provide evidence on state-wise estimates of COPD; however, there are no robust national-level prevalence estimates currently available. Data on the prevalence of COPD at the state level is available, but no review or meta-analysis has been conducted in the recent past at the country level to generate evidence on the burden of COPD among the population above 30 years in India. Also, there is a growing burden of COPD, partly due to the aging of the population [7]. COPD also develops over a decade of exposure to inhaled particles [27], which makes it crucial to estimate COPD prevalence among the Indian population aged 30 years and above. Additionally, ascertaining the risk factors for COPD would help guide projections of the disease burden at the national level and assist decision-makers in planning for service delivery that rising COPD rates will develop.

The objectives of the present review are (i) to estimate the prevalence of COPD among population above 30 years in India, (ii) to ascertain risk factors associated with COPD among the Indian population, and (iii) to compare the prevalence of COPD among population diagnosed through spirometry and nonspirometry methods.

\section{Study design}

\section{METHODS}

This systematic review and meta-analysis protocol were prepared based on Preferred Reporting Items for Systematic Reviews and Meta-Analyses [28] and Meta-analysis Of Observational Studies in Epidemiology guidelines [29]. The protocol was registered in the International Prospective Register of Systematic Reviews (PROSPERO) [30]. The results will inform the policymakers on the burden of disease along with the risk factors and shed light on the comparison of COPD diagnosed through the two methods.

\section{Data source and search strategy}

Medical Subject Headings (MeSH) and keywords will be identified through the MeSH library [31] and in consultation with the subject matter experts (technical experts for the clinical aspect of COPD and a librarian for searching the articles). Searches will be carried by two authors independently (they are both trained in evidence synthesis methods) in PubMed, Cochrane, SCOPUS, Web of Science, CINAHL, and ProQuest databases within a specified timeframe (2000 and 2020). The rationale behind the search from 2000 to 2020 is the finding from a meta-analysis, which reported that the search including articles from past 20 years will not result in a loss of any pertinent studies [32]. The language limit will be applied and only articles published in English will be considered for inclusion. A comprehensive search strategy will be designed to identify all relevant articles in India (Table 1). The reference list of all included studies will be scanned for any relevant literature.

\section{Study selection: PICOS \\ Population}

We will focus on peer-reviewed research articles based on Indian population aged 30 years and above. Studies whose target population is individuals with co-morbidities will also be excluded.

\section{Intervention and comparison}

No interventions and comparators are considered in this systematic review as we are estimating the prevalence of COPD along with the associated risk factors.

\section{Outcome}

We will focus on the prevalence of COPD among population above 30 years in India and the risk factors associated with COPD.

\section{Outcome measures}

We will search for confirmed diagnosis of COPD using spirometry where post bronchodilator forced expiratory volume (FEV1) to forced vital capacity (FEV1/FVC) is $<0.7$ and through clinical diagnosis of COPD.

\section{Study design}

Cross-sectional and cohort studies conducted among Indian population aged above 30 years reporting prevalence of COPD and (or) risk factors for COPD will be included. Studies reporting prevalence of other respiratory disorders such as asthma or studies investigating the effectiveness of therapeutic or pharmacological interventions in COPD will be excluded. Studies that are reviews, meta-analysis, commentaries, qualitative studies, and conference abstracts will also be excluded.

\section{SCREENING}

All articles identified will be subjected to screening for inclusion or exclusion against the eligibility criteria using Rayyan.qcri.org software [33]. Title and abstract screening followed by full-text screening will be done independently by two reviewers (PN, AM). Reasons for exclusion will be recorded at the screening stage. Any disagreement between the reviewers at any stage of screening will be arbitrated by a third independent reviewer to reach a consensus (MR). A PRISMA chart will be generated to illustrate this study selection process [28]. 


\section{DATA EXTRACTION AND QUALITY ASSESSMENT}

Data will be extracted independently by two reviewers using the standardized, pre-tested data extraction form on Microsoft excel 2007. Any disagreements will be resolved by discussion and consensus among the authors. The data extraction form will be developed by the authors, with technical expertise from senior reviewers, subject and clinical experts, and statisticians. The form will be pilot tested on $5 \%$ of included studies to ensure that it adequately facilitates the collection of all necessary information required for an effective meta-analysis. Data will be extracted under the following major domains: $(i)$ study characteristics, (ii) methodological characteristics, (iii) study definitions, (iv) factors identified (e.g., disease risk factors like smoking status, previous exposure of smoking, type of data, a measure of association calculated), and $(v)$ other important information such as the definition of exposure and outcome, mechanisms for the ascertainment of exposure and outcome, and controlled confounders controlled. If the study setting (urban, rural, or occupational) is not clearly defined by the authors then it will be considered as a mixed setting. Statistical analysis will be performed using Stata (V-13.1) [34]. The prevalence of COPD will be independently evaluated from spirometric and nonspirometric sets of studies as described by Adeloye et al. [35].

\section{ASSESSING THE QUALITY OF REPORTING}

Reporting quality of the studies will be assessed using the Strengthening the Reporting of Observational Studies in Epidemiology (STROBE) Statement [36]. The New Castle Ottawa Scale will be used, and the appraisal outcome will be discussed using narrative synopsis [37].

\section{PLAN FOR META-ANALYSIS}

As there is an availability of data at the state level and a shortage of evidence on COPD disease burden at the national level, we propose conducting a quantitative evaluation for evidence generation through this meta-analysis. A meta-analysis will be performed only if the studies are homogeneous and provide appropriate data required for meta-analysis. The random-effects model will be used for meta-analysis as the included studies are mostly observational. Heterogeneity will be determined by the $\chi^{2}$ statistic and $\mathrm{I}^{2}$ statistic. Heterogeneity will be considered for $\mathrm{I}^{2}>$ $50 \%$. If heterogeneity is observed, then a random-effect model will be used for meta-analysis. Results will be presented in the form of a forest plot. Pooled estimate with $95 \%$ confidence interval, $P$ value of $\chi^{2}$, and value of $\mathrm{I}^{2}$ statistic will be reported. Meta-analysis will be performed in STATA 13.1 statistical package.

\section{DISCUSSION}

This systematic review and meta-analysis will report the variation of COPD prevalence and incidence among the population aged 30 years and above in India. The methodology used in this systematic review and meta-analysis will ensure a robust knowledge synthesis of available data. This review will generate evidence for relevant stakeholders and policymakers, thereby informing policy development and program priorities for COPD in India for decision making.

\section{DISSEMINATION}

The results from the study will be disseminated to the relevant stakeholders through workshops, plain language summaries, and scientific publications.

\section{DISCLOSURES}

\section{Acknowledgments}

The authors thank Mr. Theophilus Lakiang (Independent Researcher, India) for providing valuable insights to revise the manuscript.

\section{Author contributions}

The idea for the review was generated by MPR, AV, and PN. NG, PN, $\mathrm{AM}, \mathrm{AV}$, and $\mathrm{R}$ were involved in the literature search. All the authors were involved in writing the manuscript and in the critical revision of the final manuscript.

\section{Funding}

The authors did not receive support from any organization for the submitted work.

\section{Conflict of interest}

The authors declare that they have no conflict of interest. All authors have completed the ICMJE uniform disclosure form at www.icmje.org/ coi_disclosure.pdf and declare: no financial relationships with any organizations that might have an interest in the submitted work in the previous 3 years; no other relationships or activities that could appear to have influenced the submitted work.

\section{Ethics approval}

This systematic review did not require ethical approval as it does not include human participants directly or indirectly.

\section{REFERENCES}

1. World Health. Global burden of disease study 2017. Lancet [Internet] 2017;1-7. Available at: http://ghdx.healthdata.org/gbd-2017 [Accessed 14 August 2020]

2. Alvar A, Decramer M, Frith P. Global initiative for chronic obstructive lung disease. Pocket guide to COPD diagnosis, management and prevention [Internet]. 2019. Available at: www.goldcopd.org [Accessed 14 August 2020]

3. Soriano JB, Abajobir AA, Abate KH, et al. Global, regional, and national deaths, prevalence, disability-adjusted life years, and years lived with disability for chronic obstructive pulmonary disease and asthma, 1990-2015: a systematic analysis for the Global Burden of Disease Study 2015. Lancet Respir Med 2017;5(9):691-706. doi: 10.1016/S2213-2600(17) 30293-X

4. Halbert RJ, Natoli JL, Gano A, Badamgarav E, Buist AS, Mannino DM. Global burden of COPD: systematic review and meta-analysis. Eur Respir J 2006;28(3):523-32. doi: 10.1183/09031936.06.00124605.

5. Adeloye D, Chua S, Lee C, et al. Global and regional estimates of COPD prevalence: systematic review and meta-analysis. J Glob Health 2015;5(2). doi: $10.7189 /$ jogh.05.020415.

6. World Health Organizaion. DALYs / YLDs definition [Internet]. Available at: https://www.who.int/mental_health/management/depression/daly/en/ [Accessed 14 August 2020]

7. Zamzam MA, Azab NY, El Wahsh RA, Ragab AZ, Allam EM. Quality of life in COPD patients. Respir Circ. 2002;50(3):241-5. doi: 10.1016/j. ejcdt.2012.08.012.

8. Ahmed MS, Neyaz AN, Aslami A. Health-related quality of life of chronic obstructive pulmonary disease patients: results from a community based cross-sectional study in Aligarh, Uttar Pradesh, India. Lung India 2016;33(2):148-53. doi: 10.4103/0970-2113.177438.

9. Patel JG, Coutinho AD, Lunacsek OE, Dalal AA. COPD affects worker productivity and health care costs. Int J COPD 2018;13:2301-11. doi: 10.2147/COPD.S163795.

10. Grigsby M, Siddharthan T, Chowdhury MAH, et al. Socioeconomic status and COPD among low-and middle-income countries. Int J COPD 2016;11(1):2497-507. doi: 10.2147/COPD.S111145.

11. World Health Organization. Chronic obstructive pulmonary disease (COPD). 2017. Available at: https://www.who.int/news-room/factsheets/detail/chronic-obstructive-pulmonary-disease-(copd) [Accessed 15 August 2020]

12. Government of India. National portal of India [Internet]. National Informatics Centre (NIC). Available at: https://www.india.gov.in/indiaglance/profile [Accessed 15 August 2020]

13. Salvi S, Kumar GA, Dhaliwal RS, et al. The burden of chronic respiratory diseases and their heterogeneity across the states of India: the Global Burden of Disease Study 1990-2016. Lancet Glob Health 2018;6(12): e1363-74.

14. Institute for Health Metrics and Evaluation. India [Internet]. India; 2020. Available at: http://www.healthdata.org/india [Accessed 16 August 2020]

15. MFHI, ICMR, IHME. India: health of the nation's states. Government Report. 2017. p. 70-5.

16. Lakiang T, Nair NS, Ramaswamy A, Singhal U, Özdedeoglu Ö. Economic impact of chronic obstructive pulmonary disease: a cross-sectional study at teaching hospital in South India. J Fam Med Prim Care [Internet] 2017;6(2):169-70. Available at: http://www.jfmpc.com/article.asp? issn $=2249-4863 ;$ year $=2017 ;$ volume $=6 ;$ issue $=1 ;$ spage $=169 ;$ epage $=170$; aulast=Faizi [Accessed 17 August 2020] 
17. Londhe J, Mudliar K, Powar K, et al. Direct and indirect costs of COPD treatment in Pune city, India. Eur Respir J [Internet] 2019 Sep 28;54(suppl 63):PA1967. Available at: http://erj.ersjournals.com/content/54/suppl_63/PA1967.abstract [Accessed 17 August 2020]

18. Mahmood T, Singh RK, Kant S, Shukla AD, Chandra A, Srivastava RK. Prevalence and etiological profile of chronic obstructive pulmonary disease in nonsmokers. Lung India 2017;34(2):122-6. doi: 10.4103/09702113.201298.

19. Parasuramalu BG, Huliraj N, Prashanth Kumar SP, Gangaboraiah, Ramesh Masthi NR, Srinivasa Babu CR. Prevalence of chronic obstructive pulmonary disease and its association with tobacco smoking and environmental tobacco smoke exposure among rural population. Indian J Public Health 2014;58(1):45-9. doi: 10.4103/0019-557X.128166.

20. Sinha B, Singla R, Chowdhury R. An epidemiological profile of chronic obstructive pulmonary disease: a community-based study in Delhi. J Postgrad Med 2017;63(1):29-35. doi: 10.4103/0022-3859.194200.

21. Jindal SK, Aggarwal AN, Gupta D. A review of population studies from India to estimate national burden of chronic obstructive pulmonary disease and its association with smoking. Indian J Chest Dis Allied Sci [Internet] 2001;43(3):139-47. Available at: https://pubmed.ncbi.nlm. nih.gov/11529432/ [Accessed 17 August 2020]

22. Dutta S, Deshmukh PR. Prevalence and determinants of self-reported chronic bronchitis among women in rural Central India. Med J Armed Forces India 2015 Jan;71(1):48-52. doi: 10.1016/j.mjafi.2014.10.002.

23. Viswanathan K, Rakesh PS, Balakrishnan S, Shanavas A, Dharman V. Prevalence of chronic respiratory diseases from a rural area in Kerala, southern India. Indian J Tuberc 2018 Jan;65(1):48-51. doi: 10.1016/j. ijtb.2017.01.010.

24. WHO. Global surveillance, prevention and control of chronic respiratory diseases: a comprehensive approach [Internet]. 2007. Available at: https://www.who.int/gard/publications/GARD_Manual/en/ [Accessed 17 August 2020]

25. Salvi S, Barnes PJ. Is exposure to biomass smoke the biggest risk factor for COPD globally? Chest 2010;138(1):3-6. doi: 10.1378/chest.10-0645.

26. De Koning HW, Smith KR, Last JM. Biomass fuel combustion and health. Bull World Health Organ 1985;63(1):11-26.

27. Buist AS, McBurnie MA, Vollmer WM, et al. International variation in the prevalence of COPD (the BOLD Study): a population - based prevalence study. Lancet 2015;370(9589):741-50. doi: 10.1016/S0140-6736(07)61377-4.
28. Liberati A, Altman DG, Tetzlaff J, et al. The PRISMA statement for reporting systematic reviews and meta-analyses of studies that evaluate healthcare interventions: explanation and elaboration. BMJ 2009;339. doi: 10.1136/bmj.b2700.

29. Stroup DF, Berlin JA, Morton SC, et al. Meta-analysis of observational studies in epidemiology: a proposal for reporting. Meta-analysis Of Observational Studies in Epidemiology (MOOSE) group. JAMA 2000;283(15):2008-12. doi: 10.1001/jama.283.15.2008.

30. Gudi N, Mahmood A, Roy MP, Ravishankar N, Verma A, Nayak P. Burden of COPD among population above 30 years in India: systematic review and meta-analysis [Internet]. PROSPERO 2020. Available at: https://www.crd.york.ac.uk/prospero/display_record.php?ID= CRD42020201377 [Accessed 19 August 2020]

31. National Library of Medicine. Medical subject headings [Internet]. National Institute of Health; 2020. Available at: https://www.nlm.nih. gov/mesh/meshhome.html [Accessed 19 August 2020]

32. Marshall IJ, Marshall R, Wallace BC, Brassey J, Thomas J. Rapid reviews may produce different results to systematic reviews: a meta-epidemiological study. J Clin Epidemiol 2019 May;109:30-41. doi: 10.1016/j. jclinepi.2018.12.015.

33. Ouzzani M, Hammady H, Fedorowicz Z, Elmagarmid A. Rayyan-a web and mobile app for systematic reviews. Syst Rev 2016;5(1):1-10. doi: 10.1186/s13643-016-0384-4.

34. Stata Statistical Software: release 13. College Station, TX: StataCorp; 2013.

35. Adeloye D, Basquill C, Papana A, Chan KY, Rudan I, Campbell H. An estimate of the prevalence of COPD in Africa: a systematic analysis. COPD J Chronic Obstr Pulm Dis 2015;12(1):71-81. doi: 10.3109/ 15412555.2014.908834.

36. von Elm E, Altman DG, Egger M, Pocock SJ, Gøtzsche PC, Vandenbroucke JP. The Strengthening the Reporting of Observational Studies in Epidemiology (STROBE) statement: guidelines for reporting observational studies. J Clin Epidemiol 2008;61(4):344-9. doi: 10.1016/j. jclinepi.2007.11.008.

37. Wells G, Shea B, O'Connell D, Peterson J, Welch V, Losos M TP. The Newcastle-Ottawa Scale (NOS) for assessing the quality of nonrandomised studies in meta-analyses. Vis Commun Q [Internet] 2013. Available at: http://www.ohri.ca/programs/clinical_epidemiology/ oxford.asp [Accessed 19 August 2020] 